EPJ Web of Conferences 20, 03006 (2012)

DOI: $10.1051 /$ epjconf/20122003006

(C) Owned by the authors, published by EDP Sciences, 2012

\title{
QCD Phase Diagram with Imaginary Chemical Potential
}

\author{
Keitaro Nagata ${ }^{a}$ and Atsushi Nakamura \\ Research Institute for Information Science and Education, Hiroshima University, Higashi-Hiroshima \\ 739-8527 Japan
}

\begin{abstract}
We report our recent results on the QCD phase diagram obtained from the lattice QCD simulation. The location of the phase boundary between hadronic and QGP phases in the two-flavor QCD phase diagram is investigated. The imaginary chemical potential approach is employed, which is based on Monte Carlo simulations of the QCD with imaginary chemical potential and analytic continuation to the real chemical potential region.
\end{abstract}

\section{Introduction}

The QCD phase diagram, which illustrates states of matter formed in terms of the strong interaction at a temperature and chemical potential, has been of prime interest in recent physics covering particle physics, hadron/nuclear physics and astrophysics. On top of ordinary nuclear matter and hot or dense matter such as QGP or compact stars, a very rich structure has been predicted in the QCD phase diagram using many phenomenological studies. Thoroughgoing analyses of heavy ion data show that we are sweeping finite temperature and density regions. See Ref. [1].

First-principle calculations based on QCD are now highly called. If such calculations would be at our hand, their outcomes are also very valuable for many research fields: high energy heavy ion collisions, the high density interior of neutron stars and the last stages of the star evolution. Needless to say, the inside of nucleus is also a baryon rich environment, and lots of contributions to nuclear physics could be expected.

Because QCD is non-perturbative in most regions of the QCD phase diagram, one is forced to use the lattice QCD in order to obtain a quantitative understanding. The lattice QCD is expected to provide reliable information on the phase structure based on QCD. Indeed, recently, there have been many active quantitative investigations about the finite temperature QCD [2,3].

On the other hand, simulations of systems with non-zero quark chemical potential $\mu$ have been a long challenge for the lattice QCD because of the notorious sign problem. In the lattice QCD, a fermionic determinant $\operatorname{det} \Delta(\mu)$ is used as a probability in a Monte Carlo method. The introduction of non-zero $\mu$ makes det $\Delta(\mu)$ complex, and therefore leads to the breakdown of the stochastic part of the lattice QCD. Despite of the severe sign problem, several approaches have been proposed to study the QCD with nonzero $\mu$, where the location of the phase boundary and critical endpoint, and the determination of EoS have been extensively investigated. See e.g. [4,5].

One idea is to perform simulations in systems with an imaginary chemical potential, where the sign problem is absent. The phase diagram in the imaginary chemical potential region is connected to that in the real chemical potential region, i.e., ordinary QCD phase diagram, owing to the analytic continuation. In addition, HMC algorithms are available without any truncation for the quark determinant containing imaginary chemical potential. Numerical costs are relatively small compared to approaches requiring the direct evaluation of the quark determinant. Hence, the lattice QCD with imaginary chemical potential is one of the standard technique and has been studied by using staggered fermions with

\footnotetext{
a e-mail: kngt@hiroshima-u.ac.jp
} permits unrestricted use, distribution, and reproduction in any noncommercial medium, provided the original work is properly cited. 
two flavor [6-8], three flavor [9], four flavor [10-13] in 2-color QCD and finite isospin QCD [14, 15], Wilson fermions with two flavor [16] and clover-improved Wilson fermions with two flavor [17]. In addition, data obtained in such a simulation are also useful the matching of phenomenological models such as Polyakov loop extended Nambu-Jona-Lasinio(PNJL) models with the lattice QCD [19-22].

Recently, we have studied the two-flavor QCD phase diagram using the lattice QCD simulations $[18,17]$. We have used the imaginary chemical potential approach to avoid the sign problem. Here, we report our results on the study of the QCD phase diagram.

\section{QCD with Imaginary Chemical Potential}

Let chemical potential complex $\mu=\mu_{R}+i \mu_{I}\left(\mu \in \mathbb{C}, \mu_{R}, \mu_{I} \in \mathbb{R}\right)$. Fermion determinants satisfy a relation

$$
\Delta(\mu)^{\dagger}=\gamma_{5} \Delta\left(-\mu^{*}\right) \gamma_{5},
$$

where $\Delta(\mu)$ is a quark matrix. It is straightforward from Eq. (1) to obtain $(\operatorname{det} \Delta(\mu))^{*}=\operatorname{det} \Delta\left(-\mu^{*}\right)$. This implies that $\operatorname{det} \Delta(\mu)$ is complex for a real chemical potential $\mu=\mu_{R}$, which causes the sign problem. On the other hand, one can easily prove $\operatorname{det} \Delta(\mu)$ is real for a pure imaginary chemical potential $\mu=i \mu_{I}$. The sign problem does not occur in this case, and Monte Carlo methods are available.
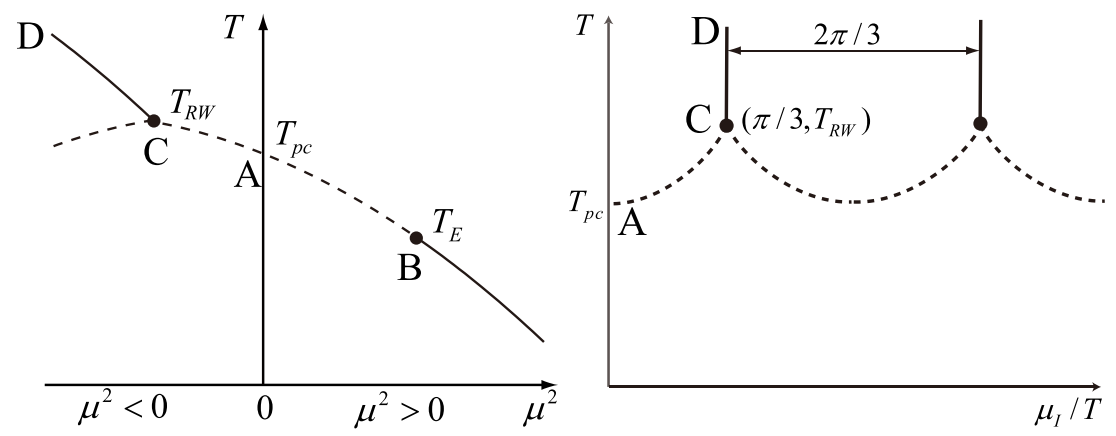

Fig. 1. Schematic figures for the $N_{f}=2$ QCD phase diagram in the $\left(\mu^{2}, T\right)$ plane (left) and $\left(\mu_{I} / T, T\right)$ plane (right). A : Pseudo-critical point at $\mu=0$. B : Critical endpoint. C : RobergeWeiss endpoint. AB : Pseudo-critical line. AC : Extension of the line $\mathrm{AB}$ into the imaginary chemical potential plane. CD : Roberge-Weiss phase transition line $\mu_{I} / T=\pi / 3$. In the right panel, larger $\mu_{I} / T$ region of the phase diagram is obtained from the RW periodicity.

Two characteristics of the $\mu^{2} \leq 0$ region are so-called Roberge-Weiss(RW) phase transition and Roberge-Weiss(RW) periodicity [23]. The QCD grand partition function has a periodicity with a period $2 \pi / N_{c}$ as

$$
Z\left(\frac{\mu_{I}}{T}\right)=Z\left(\frac{\mu_{I}}{T}+\frac{2 \pi k}{N_{c}}\right),
$$

where $k$ is an integer. Furthermore, Roberge and Weiss showed from a perturbative analysis the existence of a first-order phase transition on the line $\mu_{I} / T=\pi / N_{c}$, and from a strong coupling analysis the absence of such a transition at low temperatures. Because the RW phase transition occurs at high temperatures but does not at low temperatures, it may have an endpoint at a temperature $T_{R W}$ on the line $\mu_{I} / T=\pi / 3$. The order of the point has been extensively investigated in Ref. [7,9] and the quark-mass dependence is found: first order for small and large quark masses and second order for an intermediate quark masses. 
The left panel of Fig. 1 shows an expected phase diagram, where we employ the $\left(\mu^{2}, T\right)$ plane containing both the real $\left(\mu^{2} \geq 0, \mu=\mu_{R}\right)$ and imaginary $\left(\mu^{2} \leq 0, \mu=i \mu_{I}\right)$ regions. Even if $\mu^{2} \leq 0$, it is expected quark-gluon-plasma(QGP) and hadronic phases exist at high and low temperatures, respectively. The two phases are separated by the pseudocritical line for the deconfinement crossover, which is an extension from the $\mu^{2} \geq 0$ region. The absolute value of the Polyakov loop is often employed to identify confinement/deconfinement phase, although it is not a real order parameter because of the crossover nature of the transition. The features in the $\mu^{2} \leq 0$ region are well manifested in the $\left(\mu_{I} / T, T\right)$-phase diagram, see the right panel of Fig. 1.

\section{Result}

\subsection{Set Up}

We employ a clover-improved Wilson fermion action of two-flavors and a renormalization-group improved gauge action. The clover-improved Wilson fermion action is given by

$$
\begin{aligned}
& \Delta(x, y)=\delta_{x, x^{\prime}}-\kappa \sum_{i=1}^{3}\left[\left(1-\gamma_{i}\right) U_{i}(x) \delta_{x^{\prime}, x+\hat{i}}+\left(1+\gamma_{i}\right) U_{i}^{\dagger}\left(x^{\prime}\right) \delta_{x^{\prime}, x-\hat{i}}\right] \\
& -\kappa\left[e^{+\mu}\left(1-\gamma_{4}\right) U_{4}(x) \delta_{x^{\prime}, x+\hat{4}}+e^{-\mu}\left(1+\gamma_{4}\right) U_{4}^{\dagger}\left(x^{\prime}\right) \delta_{x^{\prime}, x-\hat{4}}\right]-\kappa C_{S W} \delta_{x, x^{\prime}} \sum_{\mu \leq v} \sigma_{\mu \nu} F_{\mu \nu} .
\end{aligned}
$$

Here $\mu$ is the quark chemical potential in lattice unit, which is introduced to the temporal part of link variables.

In order to scan the phase diagram, simulations were done for more than 150 points on the $\left(\mu_{I}, \beta\right)$ plane in the domain $0 \leq \mu_{I} \leq 0.28800$ and $1.79 \leq \beta \leq 2.0$. All the simulations were performed on a $N_{s}^{3} \times N_{t}=8^{3} \times 4$ lattice. The RW phase transition line in the present setup is given by $\mu_{I}=\pi / 12 \sim$ 0.2618 . The value of the hopping parameter $\kappa$ were determined for each value of $\beta$ according to a line of the constant physics with $m_{P S} / m_{V}=0.8$ obtained in Ref. [25].
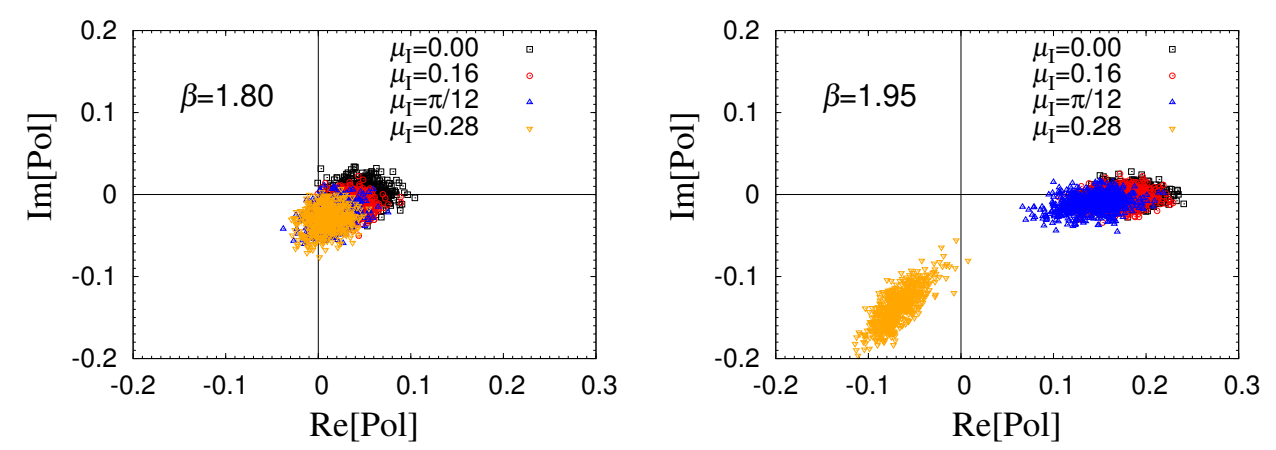

Fig. 2. Scatter plots of the Polyakov loop. Left : $\beta=1.80\left(T<T_{p c}\right)$. Right $: \beta=1.95$ ( $\left.T>T_{R W}\right)$.

Scatter plots of the Polyakov loop in the complex plane are shown in Fig. 2, where we choose two typical cases $\beta=1.80$ for the hadronic phase and $\beta=1.95$ for the QGP phase. At low temperatures, the Polyakov loop is small in magnitude for any $\mu_{I}$ and continuously changes in a clockwise direction as increasing $\mu_{I}$. On the other hand, at high temperatures, the Polyakov loop grows to $0.2 \sim 0.3$. It stays at the real axis for $\mu_{I}<\pi / 12$ and jumps to the left-lower side at $\mu_{I}=\pi / 12$. The difference of the Polyakov loop modulus between high and low temperatures shows the deconfinement crossover, which is the curve AC in Fig. 1. The observed jump of the Polyakov loop at $\mu_{I}=\pi / 12$ is the Roberge-Weiss phase transition, which is the line CD. 


\section{EPJ Web of Conferences}

\subsection{Phase transitions and universality}

Now we discuss the properties of the phase transitions in the imaginary chemical potential region of the QCD phase diagram.
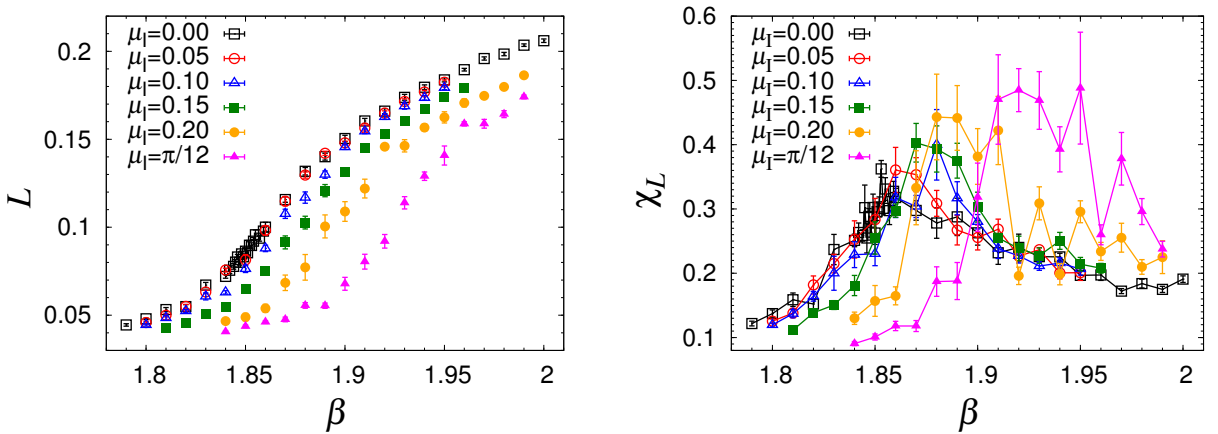

Fig. 3. The $\beta$-dependence of the Polyakov loop modulus $L$ (left) and its susceptibility $\chi_{L}$ (right) for various $\mu_{I}$.

The results of the Polyakov loop modulus $L$ and its susceptibility $\chi_{L}$ are shown in Fig. 3. Those behaviors suggest the possibility that the system undergoes the crossover with increasing $\beta$ or temperature. It is understood from the $\mu_{I}$ dependence of the peak position of $\chi_{L}$ that the pseudo-critical temperatures become higher with increasing $\mu_{I}$ until $\mu_{I}=\pi / 12$. This is consistent with the expected behavior shown in Fig. 1.

At $\mu_{I}=\pi / 12$, the system is on the RW phase transition line at high temperatures. Although the behavior of the $L$ and $\chi_{L}$ are similar, the two state signal is found for $\mu_{I}=\pi / 12$, see Fig. 6 .
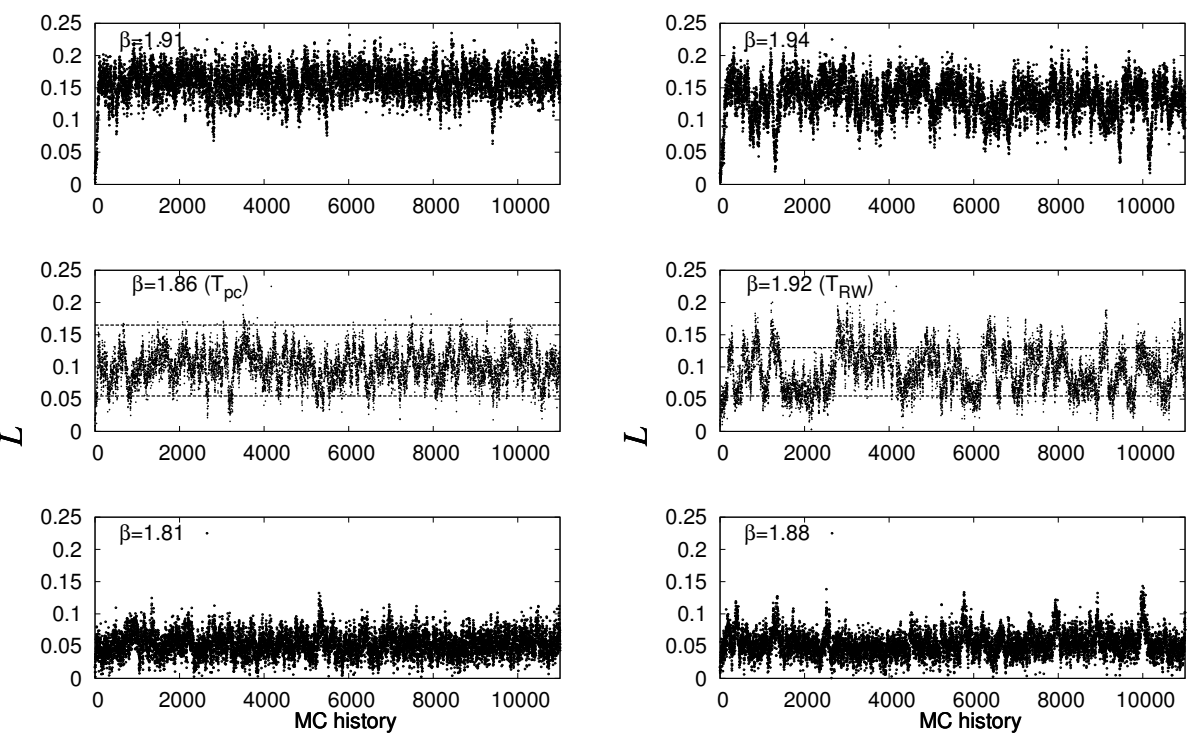

Fig. 4. Monte Carlo history of the Polyakov loop modulus at $\mu=0$ (left panels) and $\mu=\pi / 12$ (right panels). Three values of $\beta$ are chosen here. The two state signal is found in the middle of the right panels. 

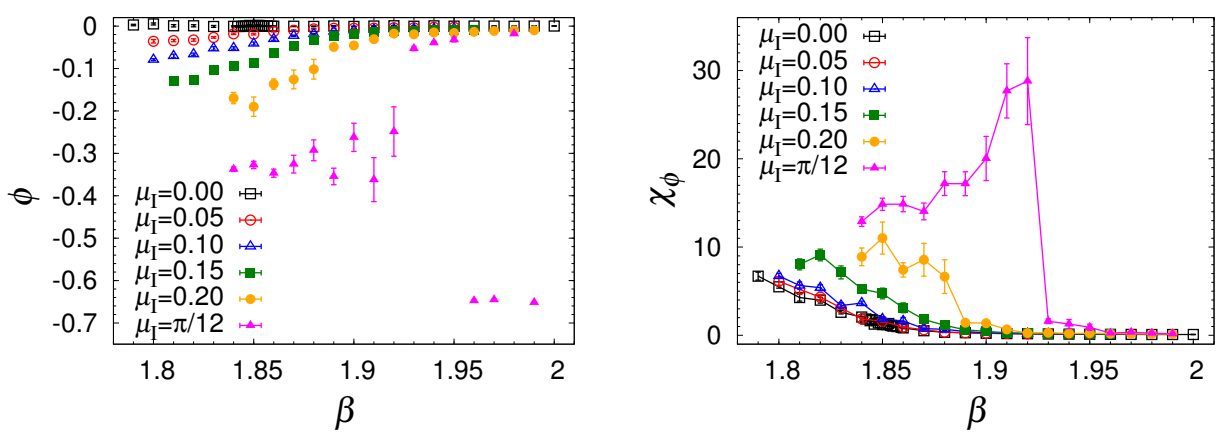

Fig. 5. The $\beta$-dependence of the Polyakov loop phase $\phi$ (left) and its susceptibility $\chi_{\phi}$ (right) for various $\mu_{I}$.
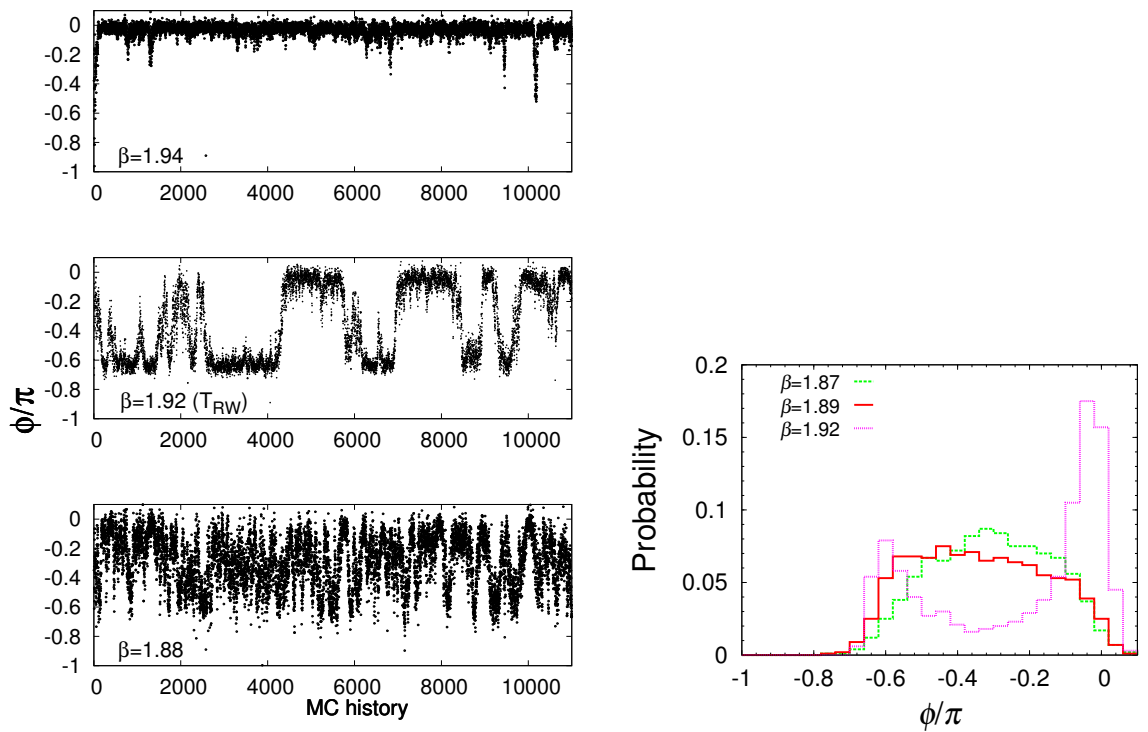

Fig. 6. Monte Carlo history of the Polyakov loop phase at $\mu_{I}=\pi / 12$ (left panels). The two state signal is found in the middle of the left panels. The histogram of $\phi$ at $\mu_{I}=\pi / 12$ for various $\beta$ (right).

It is more convenient to consider the Polyakov loop phase $\phi$ and its susceptibility $\chi_{\phi}$ in order to study the nature of the RW endpoint, which are shown in Fig. 5. For $\mu_{I}=\pi / 12, \phi$ rapidly changes near $\beta=1.92$. It is seen that for $\mu_{I}=\pi / 12$ there is one vacuum at low temperatures and are two vacua at high temperatures. The histogram and Monte Carlo history of $\phi$ at $\mu_{I}=\pi / 12$ in Fig. 6 also show this behavior. The susceptibility $\chi_{\phi}$ shows a divergent-like behavior near $\beta=1.92$ only for $\mu_{I}=\pi / 12$. These behaviors suggest the possibility that the system undergoes the second order phase transition at the RW endpoint with increasing temperature. We are now studying a finite size scaling analysis using lattice sizes $N_{s}=6,10$. Preliminary result supports that the RW endpoint is second order.

The $\mu_{I}$-dependence of $\phi$ and $\chi_{\phi}$ are shown in Fig. 7. $\phi$ is a smooth function of $\mu_{I}$ at low temperatures $(\beta=1.80-1.90)$, while $\phi$ jumps to $-2 \pi / 3$ from 0 at $\mu_{I}=\pi / 12$ at a high temperature $(\beta=1.95)$. The system undergoes the first order phase transition at $\mu_{I}=\pi / 12$ at high temperatures. Note $L$ 

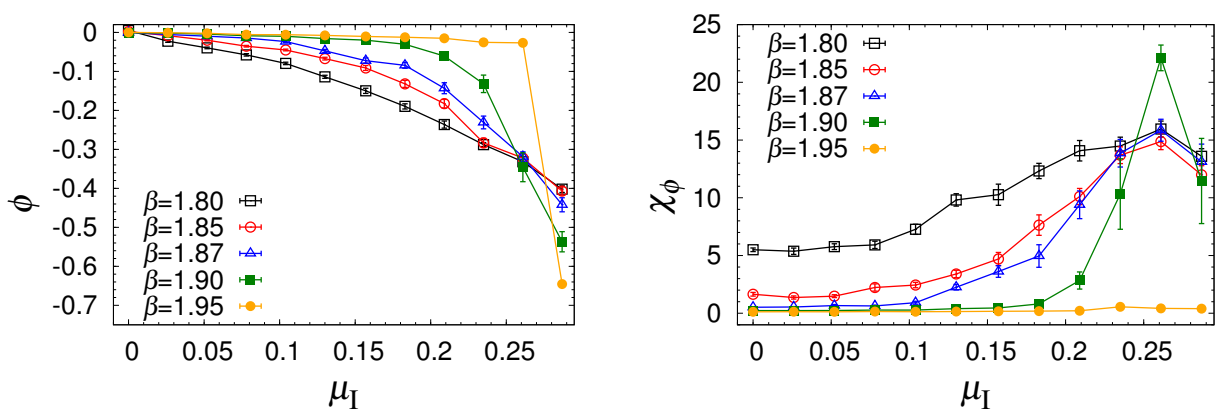

Fig. 7. The $\mu_{I}$-dependence of $\phi$ and $\chi_{\phi}$ for various $\beta$.

is periodic and $\phi$ is anti-periodic, which is caused by the periodicity of the $\mu_{I}$-dependence of the Polyakov loop [20].

Now, the properties of the QCD phase diagram with imaginary chemical potential is summarized in Fig. 8.
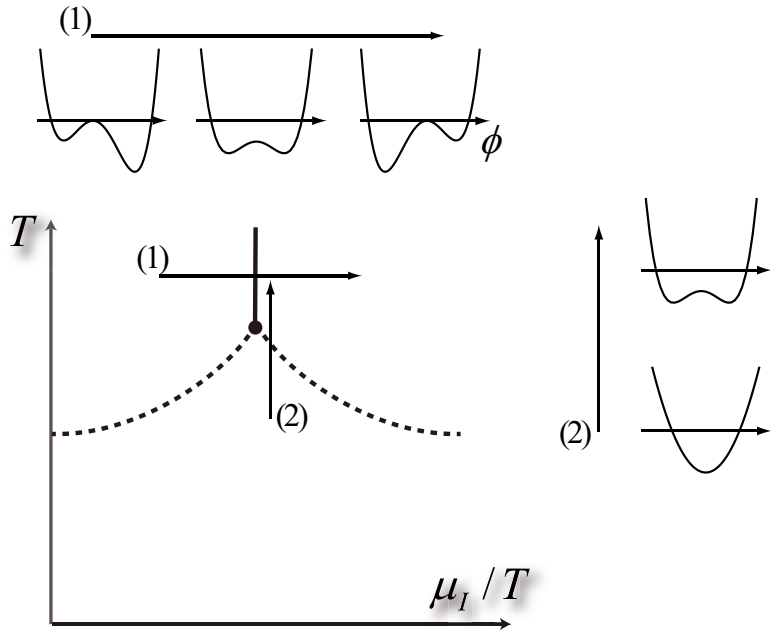

Fig. 8. Nature of the imaginary chemical potential region of the two-flavor QCD phase diagram with an intermediate quark mass obtained from the lattice QCD simulation with the cloverimproved Wilson fermion.

\subsection{Pseudocritical Line}

Next, we determine the pseudocritical line. Critical values of $\beta$ for the deconfinement crossover are obtained from the susceptibility of the Polyakov loop modulus for each $\mu_{I}$. We use the result obtained by WHOT collaboration [25] to translate $\beta$ to $T$.

We consider quadratic, quartic functions and two types of the Padé approximation $[15,14,13]$;

$$
\frac{T_{p c}}{T_{p c}^{0}}=\sum_{n} d_{n}\left(\frac{\hat{\mu}_{I}}{T_{p c}}\right)^{2 n},
$$




$$
\begin{aligned}
\frac{T_{p c}}{T_{p c}^{0}} & =d_{0} \frac{1+d_{1}\left(\hat{\mu}_{I} / T_{p c}\right)^{2}}{1+d_{2}\left(\hat{\mu}_{I} / T_{p c}\right)^{2}} \quad(\text { Padé }(\mathrm{I})), \\
\left(\frac{T_{p c}}{T_{p c}^{0}}\right)^{2} & =d_{0} \frac{1+d_{1}\left(\hat{\mu}_{I} / T_{p c}\right)^{2}}{1+d_{2}\left(\hat{\mu}_{I} / T_{p c}\right)^{2}+d_{3}\left(\hat{\mu}_{I} / T_{p c}\right)^{4}} \quad \text { (Padé (II)), }
\end{aligned}
$$

where $\mu_{I}=a \hat{\mu}_{I}$, and $\hat{\mu}_{I}$ is the imaginary chemical potential in physical unit. $T_{p c}^{0}$ and $T_{p c}$ are pseudocritical temperatures at zero and finite chemical potentials. Note that $d_{0}\left(=T_{p c} / T_{p c}(0)\right.$ at $\left.\mu=0\right)$ deviates from one with $1 \%$ because of the disagreement of $\beta_{p c}(0)$ from Ref. [25]. The result is shown in Fig. 9 (left panel). The line is almost proportional to the quadratic function at small chemical potentials, and shows the rapid increase near $\mu_{I}=\pi / 12$. We obtain the location of the RW endpoint $\beta=1.927(5)$, which corresponds to $T / T_{p c} \sim 1.15$. Except for the quadratic function, other three functions are consistent with obtained data. The quartic function suffers from large errors. The difference between two Padé approximations is found near the RW endpoint.

Obtained pseudo-critical line is analytically continued to $\mu^{2}>0$ region using $\mu_{I}^{2}=-\mu^{2}$. Th results are shown in the right panel of Fig. 9. The curvature at $\hat{\mu} / T_{p c}=0$ of a power series of $\left(\hat{\mu} / \pi T_{p c}\right)^{2}$ is $t_{2}=\pi^{2} d_{2}=0.38(12)$. The present results are slightly smaller than other studies, see e.g. Ref. [26]
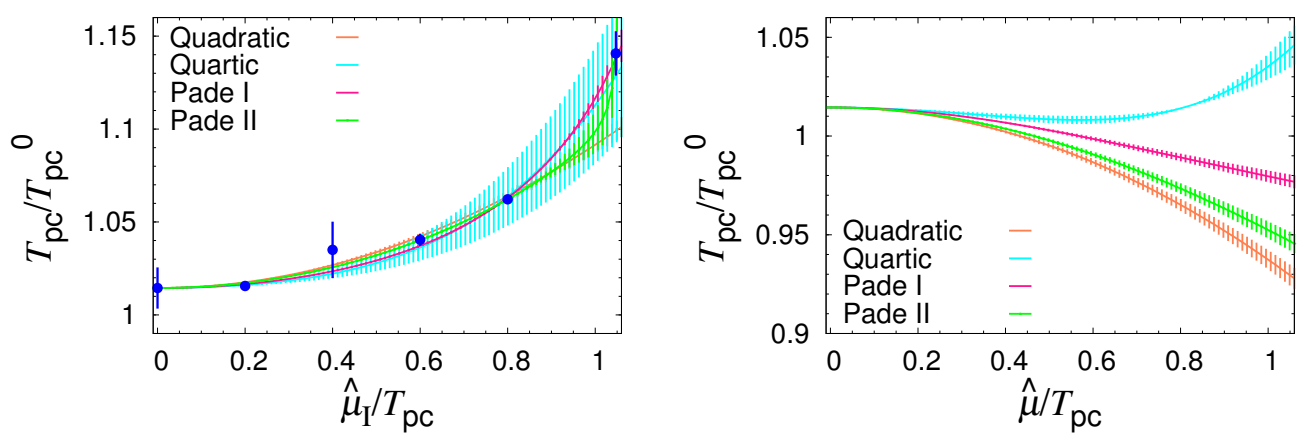

Fig. 9. The pseudo-critical line $\beta_{p c}$ in the imaginary (left panel) and real(right panel) region.

\section{Summary and outlook}

We have investigated the two-flavor QCD phase diagram using the lattice QCD simulation with the imaginary chemical potential approach. The simulation was performed on the imaginary chemical potential region. The properties of the imaginary chemical potential region of the QCD phase diagram was discussed. We have derived the pseudocritical line, and the results are analytically continued to the real chemical potential region.

The present calculation was performed with the intermediate quark mass and small lattice. The finite volume scaling analysis and quark mass-dependence analysis are necessary to confirm the present results. In particular, the order of the RW endpoint depends on the mass of the quark. The improvement on these points should be done in a future study. The finite volume scaling analysis is under progress.

\section{Acknowledgment}

We thank P. Cea, L. Cosmai, M. D’Elia, Ph. de Forcrand, H. Kouno, S. Motoki, Y. Nakagawa, A. Papa, T. Saito, Y. Sakai, T. Sasaki and M. Yahiro for discussions and comments. The simulation was performed on NEC SX-8R at RCNP, and NEC SX-9 at CMC, Osaka University, and HITACHI SR11000 
EPJ Web of Conferences

and IBM Blue Gene/L at KEK. This work was supported by Grants-in-Aid for Scientific Research 20340055 and 20105003.

\section{References}

1. A. Andronic et al., Nucl.Phys. A837, 65 (2010), arXiv:0911.4806.

2. Z. Fodor, Proceedings of XL International Symposium on Multiparticle Dynamics, Univ. Antwerp (2011).

3. C. DeTar, arXiv:1101.0208.

4. S. Muroya, A. Nakamura, C. Nonaka, and T. Takaishi, Prog.Theor.Phys. 110, 615 (2003), arXiv:hep-lat/0306031.

5. P. de Forcrand, PoS LAT2009, 010 (2009), arXiv:1005.0539.

6. P. de Forcrand and O. Philipsen, Nucl. Phys. B642, 290 (2002), arXiv:hep-lat/0205016.

7. M. D'Elia and F. Sanfilippo, Phys. Rev. D80, 111501 (2009), arXiv:0909.0254.

8. M. D'Elia and F. Sanfilippo, Phys.Rev. D80, 014502 (2009), arXiv:0904.1400.

9. P. de Forcrand and O. Philipsen, Phys.Rev.Lett. 105, 152001 (2010), arXiv:1004.3144.

10. M. D'Elia and M.-P. Lombardo, Phys.Rev. D67, 014505 (2003), arXiv:hep-lat/0209146.

11. M. D'Elia and M. P. Lombardo, Phys.Rev. D70, 074509 (2004), arXiv:hep-lat/0406012.

12. M. D’Elia, F. Di Renzo, and M. P. Lombardo, Phys.Rev. D76, 114509 (2007), arXiv:0705.3814.

13. P. Cea, L. Cosmai, M. D'Elia, and A. Papa, Phys.Rev. D81, 094502 (2010), arXiv:1004.0184.

14. P. Cea, L. Cosmai, M. D’Elia, C. Manneschi, and A. Papa, Phys.Rev. D80, 034501 (2009), arXiv:0905.1292.

15. P. Cea, L. Cosmai, M. D’Elia, and A. Papa, Phys.Rev. D77, 051501 (2008), arXiv:0712.3755.

16. L.-K. Wu, X.-Q. Luo, and H.-S. Chen, Phys. Rev. D76, 034505 (2007), arXiv:hep-lat/0611035.

17. K. Nagata, A. Nakamura, Phys. Rev. D83, 114507 (2011). [arXiv:1104.2142 [hep-lat]].

18. K. Nagata, A. Nakamura, Phys. Rev. D82, 094027 (2010). [arXiv:1009.2149 [hep-lat]].

19. Y. Sakai, K. Kashiwa, H. Kouno, and M. Yahiro, Phys. Rev. D77, 051901 (2008), arXiv:0801.0034.

20. H. Kouno, Y. Sakai, K. Kashiwa, and M. Yahiro, J. Phys. G36, 115010 (2009), arXiv:0904.0925.

21. T. Sasaki, Y. Sakai, H. Kouno, and M. Yahiro, (2011), arXiv:1105.3959, * Temporary entry*.

22. Y. Sakai, H. Kouno, T. Sasaki, and M. Yahiro, (2011), arXiv:1105.0413.

23. A. Roberge and N. Weiss, Nucl. Phys. B275, 734 (1986).

24. C. Bonati, G. Cossu, M. D’Elia, A. Di Giacomo, and C. Pica, PoS LATTICE2008, 204 (2008), arXiv:0901.3231.

25. WHOT-QCD Collaboration, S. Ejiri et al., Phys.Rev. D82, 014508 (2010), arXiv:0909.2121.

26. O. Philipsen, Prog.Theor.Phys.Suppl. 174, 206 (2008), arXiv:0808.0672. 